
\title{
25 Research Square \\ FXR-mediated inhibition of autophagy contributes to nonalcoholic fatty liver disease
}

\section{Kun Wua}

Tao Zhaoa

Christer Hogstrandc

Yi-Chuang Xua

Shi-Cheng Ling

Guang-Hui Chena

Zhi Luo

\section{Video Byte}

Keywords: lipid metabolism, RNA-seq, transcriptome, autophagy, FXR, lipotoxicity, nutrition, fat, catfish, NAFLD, nonalcoholic fatty liver disease, obesity

Posted Date: June 23rd, 2020

DOI: https://doi.org/10.21203/rs.3.rs-37693/v1

License: (c) (1) This work is licensed under a Creative Commons Attribution 4.0 International License.

Read Full License 


\section{Abstract}

Too much dietary fat can be bad for our health. Chronic high lipid intake results in the accumulation of triglycerides in the liver, causing nonalcoholic fatty liver disease (NAFLD). One contributor to NAFLD is the inhibition of a process called autophagy, where the body's cells clean up intracellular components that are damaged or no longer needed. Unfortunately, how autophagy inhibition results in NAFLD is unknown. A recent study aimed to determine what molecular pathways inhibit autophagy to cause NAFLD. Using yellow catfish as a model, they compared the effects of regular and high-lipid diets on autophagy and lipid metabolism. RNA sequencing showed that a high-fat diet altered the expression of many genes associated with lipid metabolism and autophagy. A pair of proteins, FXR and CREB, served as a switch to regulate these changes, maintaining a proper fatty acid balance and protecting cells from lipid-induced damage. Although clinical studies are needed, the results suggest that targeting the FXR/CREB axis may help to control lipid accumulation and toxicity, improving the outcome for those struggling with NAFLD. 